\title{
Ten-fold spectral resolution boosting using TEDI at the Mt. Palomar NIR Triplespec spectrograph
}

\author{
David J. Erskine $^{a}$, J. Edelstein ${ }^{b}$, P. Muirhead ${ }^{c}$, M. Muterspaugh $^{d}$, K. Covey $^{e}$, \\ D. Mondo ${ }^{b}$, A. Vanderburg ${ }^{b}$, P. Andelson ${ }^{b}$, D. Kimber ${ }^{b}$, M. Sirk ${ }^{b}$, and J. Lloyd ${ }^{e}$ \\ ${ }^{a}$ Lawrence Livermore Nat. Lab., Livermore, CA \\ ${ }^{b}$ Space Sciences Lab., Univ. of Calif., Berkeley, CA \\ ${ }^{c}$ California Institute of Technology, Pasadena, CA \\ ${ }^{d}$ Tennessee State University \\ ${ }^{e}$ Cornell Univ., Ithaca, NY
}

\begin{abstract}
An optical technique called "interferometric spectral reconstruction" (ISR) is capable of increasing a spectrograph's resolution and stability by large factors, well beyond its classical limits. We have demonstrated a 6to 11-fold increase in the Triplespec effective spectral resolution $(R=2,700)$ to achieve $R=16,000$ at $4100 \mathrm{~cm}^{-1}$ to 30,000 at $9600 \mathrm{~cm}^{-1}$ by applying special Fourier processing to a series of exposures with different delays (optical path differences) taken with the TEDI interferometer and the near-infrared Triplespec spectrograph at the Mt. Palomar Observatory 200 inch telescope. The TEDI is an externally dispersed interferometer (EDI) used for Doppler radial velocity measurements on M-stars, and now also used for ISR. The resolution improvement is observed in both stellar and telluric features simultaneously over the entire spectrograph bandwidth (0.9-2.45 $\mu \mathrm{m})$. By expanding the delay series, we anticipate achieving resolutions of $\mathrm{R}=45,000$ or more. Since the delay is not continuously scanned, the technique is advantageous for measuring time-variable phenomena or in varying conditions (e.g. planetary fly-bys). The photon limited signal to noise ratio can be 100 times better than a classic Fourier Transform Spectrometer (FTS) due to the benefit of dispersion.
\end{abstract}

Keywords: High resolution spectroscopy, Externally Dispersed Interferometry, Dispersed Fixed Delay Interferometry, Resolution Boosting, Doppler radial velocimetry, Fourier Transform Spectroscopy

\section{INTRODUCTION}

Astronomical spectrograph systems are of many types, from purely dispersive to purely interferometric (see Fig. 1). Here we demonstrate on starlight at a facility spectrograph at the Mt. Palomar Observatory the novel application to the near infrared of the hybrid spectroscopic technique called interferometric spectral reconstruction (ISR) ${ }^{3-6}$ This method multiplies the native resolution of a spectrograph by a factor of an order of magnitude or more using an interferometer in series with the spectrograph. The resolution of the reconstructed spectrum (Fig. $2 \& 3$ ) can exceed the classical limits set by pixel density, focal spot blur and slit width. The stability of the net instrument lineshape is improved by an order of magnitude or more- the resolution and detailed wavelength is now determined by the interferometer not the spectrograph. Thus performance is decoupled from the spectrograph focal spot and slit, allowing lower cost or weight spectrograph designs.

The ISR technique is performed on an externally dispersed interferometer ${ }^{3,4}$ (EDI), which is a disperser and interferometer in series. The EDI was initially developed for and has successfully been demonstrated to perform precision radial velocimetry (RV) using low resolution spectrographs. ${ }^{7-9}$ Others call this technique dispersed fixed delay interferometry (DFDI) ${ }^{10}$ and have used it to find a new exoplanet. ${ }^{11}$

Author information:

D.E.: erskine1@llnl.gov, J.E.: jerrye@ssl.berkeley.edu, P.M.: Philip Muirhead philm@astro.caltech.edu, J.L.: jpl@astro.cornell.edu

UV/Optical/IR Space Telescopes and Instruments: Innovative Technologies and Concepts V, edited by Howard A. MacEwen, James B. Breckinridge, Proc. of SPIE Vol. 8146, 81460M - ( 2011 SPIE CCC code: 0277-786X/11/\$18 - doi: 10.1117/12.892664 

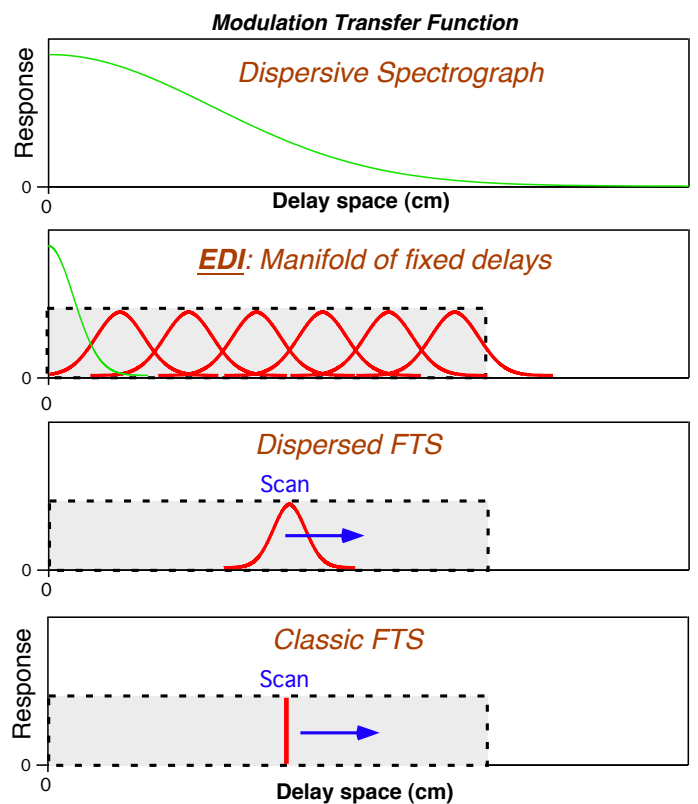

Figure 1. The information content of types of astronomical spectrograph systems, considered in frequency space, from purely dispersive (top) to purely interferometric (bottom). The EDI-ISR discussed in this paper is a hybrid dispersiveinterferometer which has many of the best characteristics of both extremes. The modulation transfer function (MTF) are plotted for each instrument, which is the Fourier transform of instrument lineshape. The horizontal axis is the Fourier space variable which is delay in $\mathrm{cm}$ or equivalently the frequency of features per wavenumber. Thin green curves are native spectrograph (nonfringing) signals, and thicker red curves are fringing-derived signals. The larger delays contain the most information about the narrow spectral components and thus are most crucial for achieving high resolution. The dashed boxes suggest how interferometers sample delay space uniformly, and thus sample the highest delays with greater sensitivity than a disperser, which falls off in sensitivity at high delays.

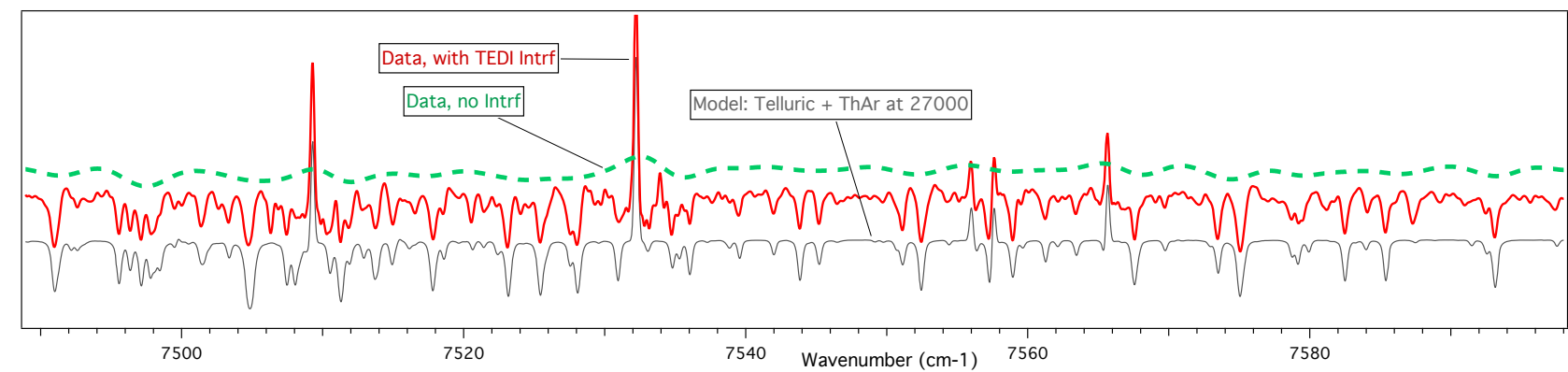

Figure 2. Demonstration of a 10 -fold boost in resolution observing telluric features mixed into spectrum of star $\kappa$ CrB along with ThAr calibration lamp emission lines. The green dashed (top) curve is the "ordinary" spectrum measured without the interference, having native resolution 2,700. It cannot resolve any of the telluric features. The red (middle) curve is the TEDI reconstructed spectrum measured with 7 contiguous delays, up to $3 \mathrm{~cm}$, and equalized to a Gaussian resolution of 27,000. The gray (bottom) curve is a model of telluric ${ }^{1}$ and $\mathrm{ThAr}^{2}$ features blurred to res of 27,000 , showing excellent agreement with ISR data. Resolution boosting occurs simultaneously across the full bandwidth of the native spectrograph. Only a small portion near $7500 \mathrm{~cm}^{-1}(1.33 \mu \mathrm{m})$ of the full Triplespec bandwidth $(0.9-2.45 \mu \mathrm{m})$ is shown here. Fig. 3 shows another section of the full spectrum. 


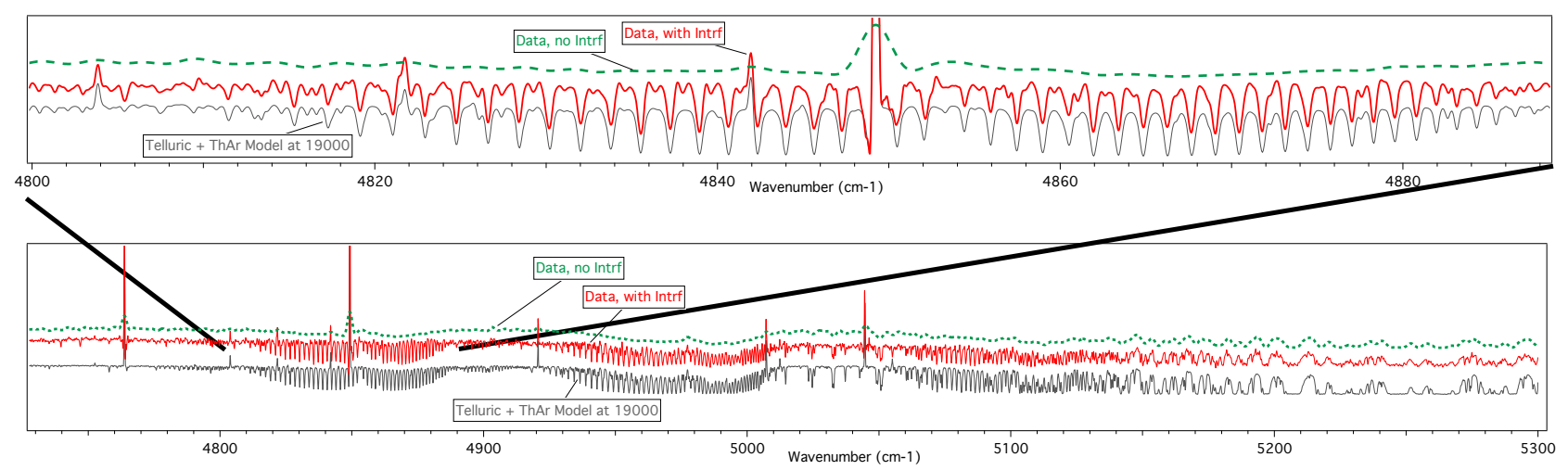

Figure 3. The ISR technique allows a wide simultaneous bandwidth only limited by the native spectrograph bandwidth. Upper graph is the zoomed in portion of a strong telluric feature. The bottom graph is half of the first order (of five) of the echelle spectrum, this order being $4100-5300 \mathrm{~cm}^{-1}(1.88-2.44 \mu \mathrm{m})$. The other orders have also been reconstructed. For this average wavenumber the reconstructed resolution is 19000, for a res boost of $7 \mathrm{x}$. Green dashed curves are without interference and cannot resolve telluric features. Red (middle) are reconstructed spectra with the interferometer, and gray (lower) are model of telluric ${ }^{1}$ and $\mathrm{ThAr}^{2}$ features blurred to 19000 resolution, showing excellent agreement.

An EDI interferometer called "TEDI" has recently successfully demonstrated precision Doppler RV of M stars in the near infrared. ${ }^{12}$ The apparatus is constrained to fit within the Cassegrain output hole of the 5meter Mt. Palomar mirror, sitting in series with the 2700 resolution TripleSpec ${ }^{13}$ NIR spectrograph (0.9-2.45 $\mu \mathrm{m})$ (Fig. 4). Using the same apparatus, we have taken additional delay data and applied different analysis to implement ISR. Thus any EDI-ISR apparatus can perform both high precision Doppler radial velocimetry and wide bandwidth high resolution spectroscopy, simply by changing which delay lengths are used and using a different data processing algorithm on the same set of primitive data. Doppler RV work will concentrate its exposure time on the highest delays, while ISR will spread exposure time across a variety of delays from near zero to a maximum value. The maximum delay divided by the wavelength sets the achievable resolution. The ability to optimize the instrument performance to different tasks is an advantage of EDI (see red vs green MTF curves in Fig. 9).

Raw fringing spectra data (Fig. 5) is taken in multiple exposures with different fixed interferometer delays, then combined and processed in the Fourier domain to reconstruct the spectrum. Unlike a classical spectrograph, the sensitivity to a feature frequency along the spectrum is not set by the focal spot size or limited by detector pixel spacing. Instead it is set by a delay (optical path difference) in an interferometer, and thus is mostly immune to environmental drifts or optical aberrations that change the spectrograph focal spot size and shape, or pupil shape. These are the bane of a classical spectroscopist.

This resolution boost occurs over the entire bandwidth of the native spectrograph with a boost factor that is proportional to the wavenumber. Thus one can achieve both high resolution and wide simultaneous bandwidth. Typical current high-resolution spectrographs achieve high resolution at the expense of simultaneous bandwidth.

The ISR technique is a dispersive-interferometric hybrid that shares some of the compactness and stablelineshape advantages of purely interferometric Fourier transform spectroscopy (FTS) but with a 100 times improved photon-limited signal to noise ratio $(\mathrm{S} / \mathrm{N})$ over the latter. The photon $\mathrm{S} / \mathrm{N}$ benefit compared to FTS goes as the square root of the number of native spectrograph resolution elements, ${ }^{14}$ which is typically $\sim 10^{4}$ for an echelle spectrograph. Since the ISR uses integrating time exposures- there is no continuously scanned delay-it is more robust to temporal fluctuations that can plague a FTS when observing a rapidly changing scene, such as spacecraft field-of-view sweeps. We have designed an EDI that uses multiple delays in parallel which can make snapshot measurements of rapidly changing phenomena (see Figs 12A \& 12B of Ref. 3).

The ISR technique is mathematically related to another hybrid, dispersed-FTS, ${ }^{15}$ but uses finer spectral channels and discrete fixed delays instead of scanning a single delay continuously over a range and reading out 

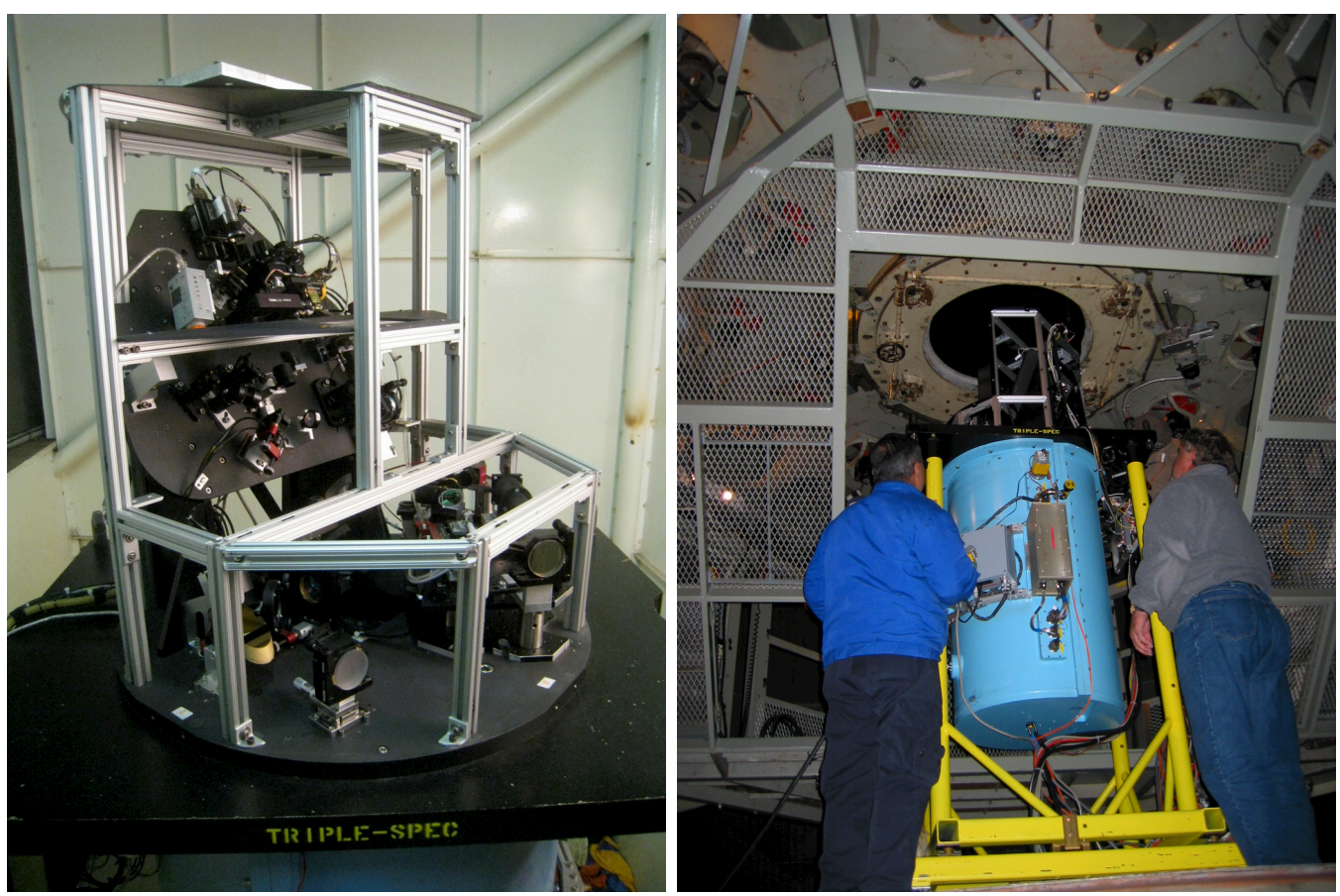

Figure 4. Photograph of the TEDI interferometer, which sits atop Cornell's TripleSpec spectrograph (blue cryogenic cylinder). The interferometer fits in the Cassegrain output cavity of the Mt. Palomar 200 inch mirror, while the TripleSpec is bolted to the bottom of the mirror. The TEDI unit captures the starlight, passes it through an interferometer, and then re-injects it to the spectrograph with a beam having same $\mathrm{f} / \#$ and focus location as original beam. (The non-interference spectra displayed in this article are with the arm in place, by summing several fringing exposures to cancel the fringing components.)

a time-dependent detector. All dispersed interferometer hybrids enjoy improved photon-limited S/N over an undispersed interferometer.

EDI-ISR can mitigate a dilemma for ground and space astronomers building the next generation of large telescopes and spaceprobes. Seeing limited ground-based spectrographs must scale in size proportional to the telescope aperture diameter and wavelength. Consequently, classical infrared high resolution spectrographs for the 30 meter class of telescopes are threatening to be prohibitively expensive. An ISR enhanced spectrograph could achieve the same resolution for a several times smaller overall size, which translates into a huge cost and weight savings. The EDI can also reduce the size of diffraction limited spectrographs. This size and weight advantage is critical for spaceborne instrumentation. There is a modest flux penalty for sending light through the additional optics of an interferometer. We believe the benefits outweigh this penalty for many applications.

Previously, the ISR technique has been demonstrated on starlight at the Lick Observatory Hamilton echelle spectrograph, using a single delay, to achieve a resolution boost of about $2 \mathrm{x}$, from 50,000 to $100,000 .{ }^{5}$ In the laboratory using an iodine cell, a boost of $6 \mathrm{x}$ from a native 25,000 to a final 140,000 has been demonstrated using four delays. ${ }^{6}$

\section{EXAMPLE RECONSTRUCTED STELLAR SPECTRA}

Here we report the first multi-delay spectral reconstruction on starlight, using 7 delays to achieve a 6- to 11-fold increase in the Triplespec effective spectral resolution, from native $\mathrm{R}=2,700$ to $\mathrm{R}=16,000$ at $4100 \mathrm{~cm}^{-1}$ to 30,000 at $9600 \mathrm{~cm}^{-1}$. Figure 2 shows a small section of spectrum having both telluric absorption and ThAr calibrant lamp emission lines near $1.3 \mu \mathrm{m}$ boosted 10x to resolution 27000. Figure 3 shows a prominent telluric feature near $2 \mu \mathrm{m}$ at res 19000 . 


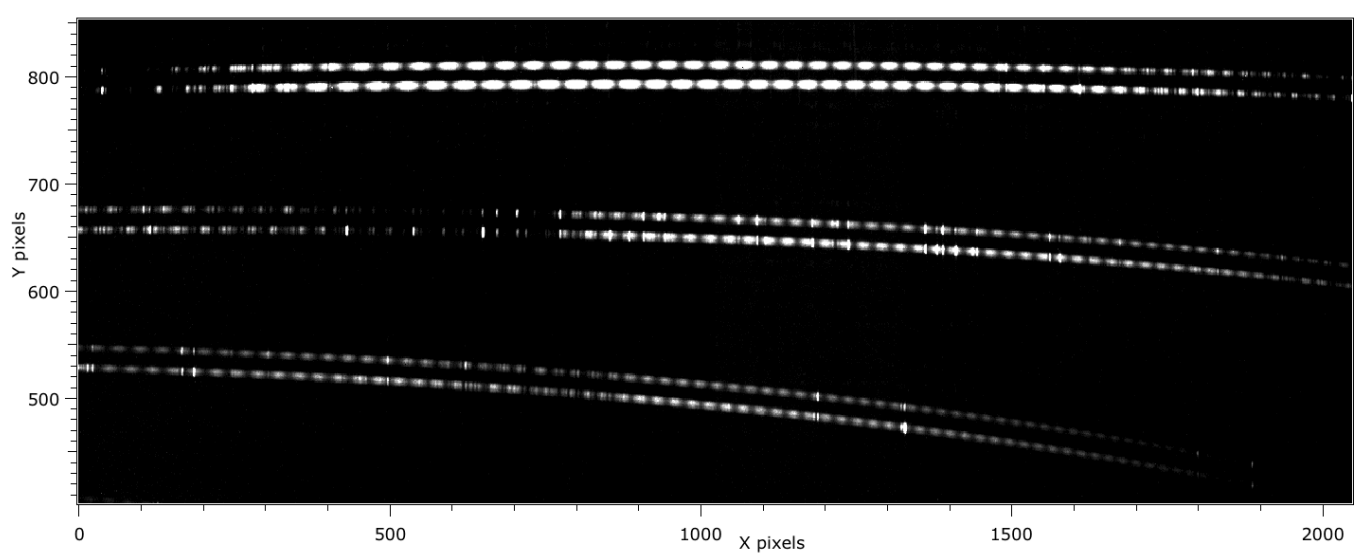

Figure 5. Three middle orders $(0.94-1.85 \mu \mathrm{m})$ out of the five of the TripleSpec echelle spectrum for star GJ 338A are shown, using a small $(0.3 \mathrm{~cm})$ delay so that the interferometer comb period is large and easily resolvable, for demonstration and alignment. Much larger delays (up to $4.6 \mathrm{~cm}$ ) are used during data-taking to create finer combs, having a period similar to the stellar features of interest. Those combs are unresolvable, but the moire patterns they create by being heterodyned against the stellar spectrum are resolvable. The moire pattern is the desired data product. The shift in moire phase (stellar vs ThAr calibrant) yields the Doppler radial velocity (for detection of exoplanets). The shape of the moire pattern vs wavenumber is Fourier processed and combined with others taken at a series of delays to yield a spectral reconstruction.

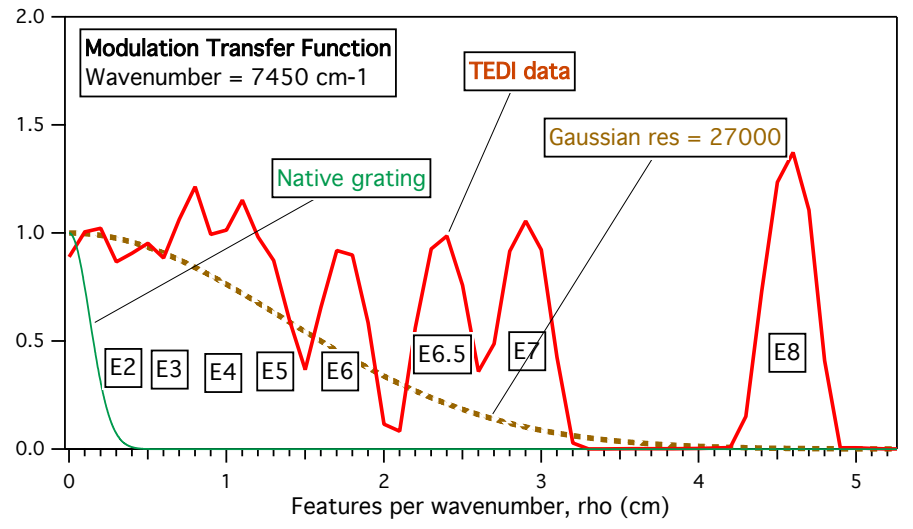

Figure 6. Net modulation transfer function (MTF) for several delays for the TEDI system near $7540 \mathrm{~cm}^{-1}(1.34 \mu \mathrm{m}) . \mathrm{A}$ MTF is the Fourier transform of the instrument lineshape. The spectrograph alone has the MTF of the thin green peak centered at the origin, whose width is proportional to its spectral resolution, in this case 2700. Including the interferometer causes a new peak to appear having same width but centered at the interferometer delay. By using a series of delays of values (E2-E7) which are contiguous, a conglomerated MTF is formed (thick red curve) which is much wider. After equalizing the peak magnitudes to better approximate a Gaussian shape (gold dash), an effective resolution of 27,000 can be achieved. By expanding the delay series (filling the gap between E7 \& E8) we anticipate achieving resolution of 45,000 at $7500 \mathrm{~cm}^{-1}$. The E6.5 delay of $2.4 \mathrm{~cm}$ replaced E1 of $0.03 \mathrm{~cm}$, to allow E7 to be contiguous with lower delays. 


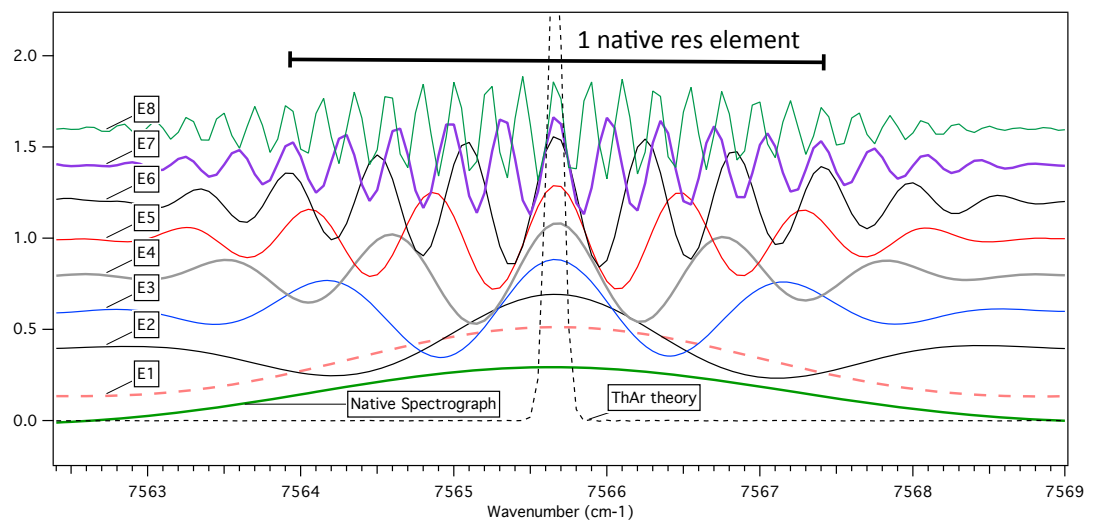

Figure 7. A set of processed data from measurment of an isolated narrow ThAr lamp line sampled by the different interferometer delays. The spectrum is reconstructed by summing processed signals from each delay channel. For each, the raw fringing signal is shifted during analysis in Fourier space to higher feature frequency by the delay in cm. This reverses the interferometer's optical heterodyning action (moiré effect) and produces wavelet-like signals shown in graph. The phases are aligned by calibrating against an isolated narrow feature, such as the theoretical ThAr lamp line shown. Amplitudes are adjusted by an equalization curve derived by comparison with an ideal Gaussian MTF having a userchosen resolution. Delay E8 is not currently used for ISR because of delay gap between E7 and E8, but is used for Doppler radial velocities.

During runs testing the TEDI radial velocity performance (which use the longest delay, $4.5 \mathrm{~cm}$ ) we took additional exposures with a contiguous set of 7 smaller delays which range from $0.3 \mathrm{~cm}$ up to $3 \mathrm{~cm}$ (Fig. 6). A contiguous coverage of delay-space is needed to produce, after equalization (multiplication to magnify or reduce), a Gaussian net instrument lineshape. This is an ideal shape for general spectroscopy because the lineshape in pixel-space lacks ringing side-lobes. With the possible addition of new thickness glass etalons (which create the field-widened angle independent delay) allowing contiguous delay coverage up to $4.5 \mathrm{~cm}$, we anticipate producing a resolution of $\sim 45000$ for a boost of about $17 \mathrm{x}$.

Figure 5 shows an example fringing spectra exposure (on 3 of 5 orders), but with an especially small delay to make the fringes more obvious for alignment purposes. Figures $7 \& 8$ shows how the spectrum is reconstructed from a set of fringing signals, one per delay. Figure 6 shows how each delay contributes a different section to the overall modulation transfer function (MTF), which we manipulate through equalization to become Gaussian. Figures $2 \& 3$ show reconstructed spectra (red curve) compared to native spectra without the interference (green curve). The native curve cannot resolve the telluric features. There is excellent agreement between the ISR result and a theoretical model of telluric ${ }^{1}$ and $\operatorname{ThAr}^{2}$ lamp lines (gray curve), which we blurred to the anticipated resolution. The resolution boost occurs over the entire original bandwidth $(0.9-2.4 \mu \mathrm{m})$.

Figure 9 shows two MTF comparing the photon limited S/N ratio between the ISR and conventional spectrograph technique, for the case where the spectrograph length is fixed, the resolution is inversely proportional to slit width, and the flux is proportional to slit width (i.e. a blurry source). A 10x resolution boost is used, the horizontal axis was calculated for $500 \mathrm{~nm}$ wavelength, and throughput loss of the interferometer is neglected. A Gaussian schedule of exposure times for seven delays is used in $\mathrm{EDI}_{1-7}$ (red curve) to best mimic the photon $\mathrm{S} / \mathrm{N}$ performance of a classic spectrograph. That is, exposure time is proportional to the square of the height of each peak shown in the Figure.

Figure 9 shows bumps in the MTF for EDI. These are equalized away in the final result but are left here for illustrative purposes. The EDI root mean square averaged $\mathrm{S} / \mathrm{N}$ is $\sqrt{2}$ lower than the classic spectrograph, but it achieves this with a much more robust instrument lineshape (Figure 10), and at much lower cost because the requirements on the focal spot size for the EDI are relaxed by an order of magnitude.

Note that we have the freedom to increase the exposure time of the longer delays to boost the $\mathrm{S} / \mathrm{N}$ in the high frequency regions that are most important for resolving and measuring narrow spectral features, such as in RV work or measuring lineshape asymmetry. For example, Fig. 9 shows the MTF called EDI $_{5-7}$ (green curve), 

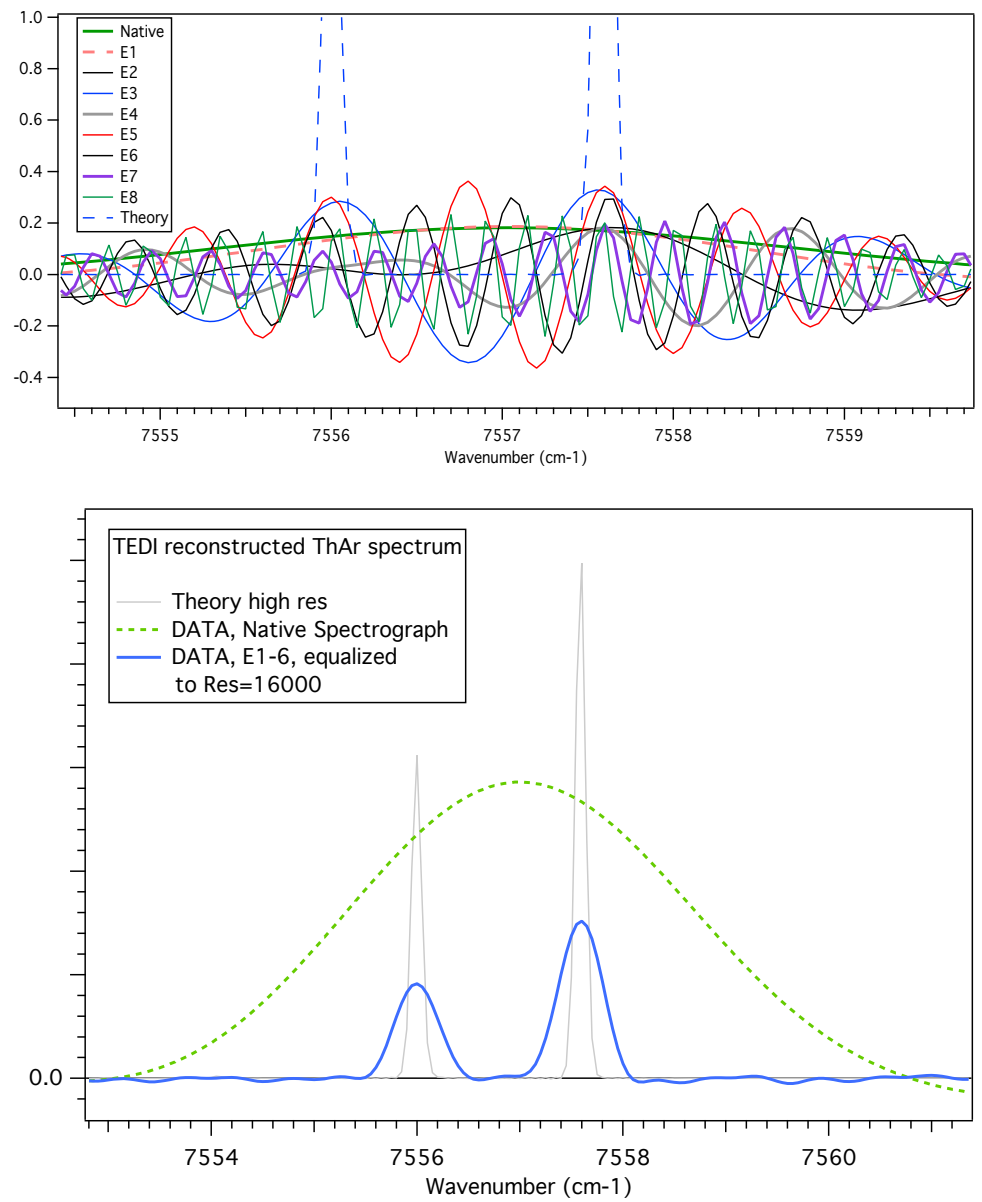

Figure 8. Reconstruction of a ThAr doublet using a neighboring single ThAr line (off graph at $7532 \mathrm{~cm}^{-1}$ ) to provide channel phase alignment information as the channels are added (top). Summed results shown in bottom graph. The native grating (green dash) cannot resolve the doublet (gray, hi res theory). The TEDI reconstructed spectrum (solid blue curve) equalized to res $=16000$ exhibits very little ringing and fully resolves the doublet. Since each channel's fringes are produced from the interferometer and not the disperser (except a broad $4 \mathrm{~cm}^{-1}$ envelope), the precision of the result does not depend on the native spectrograph or details of its slit, focal blur, pixel spacing or beamshape. 


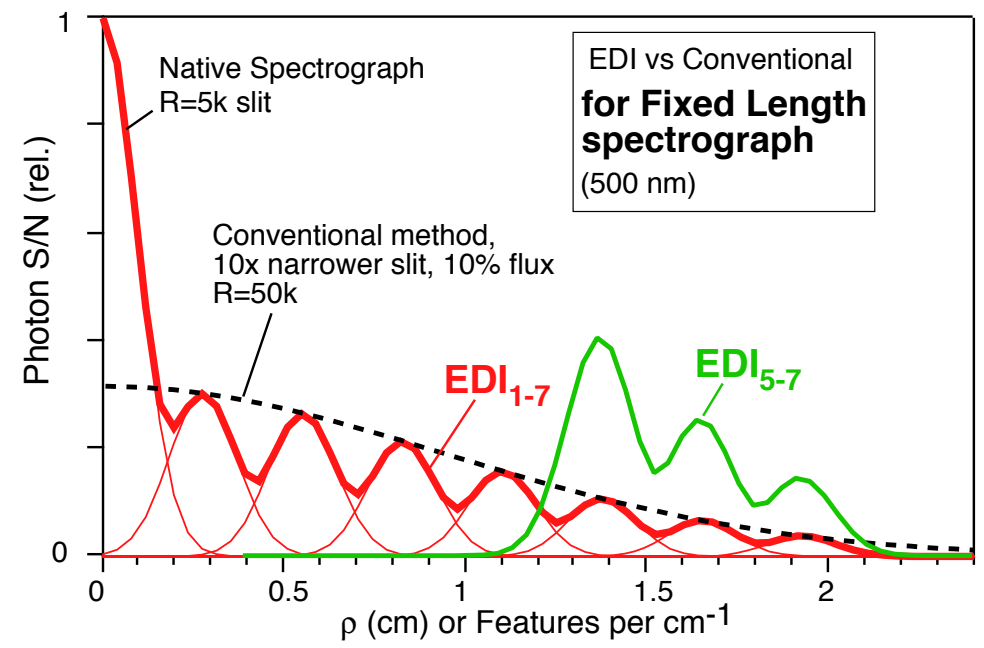

Figure 9. Comparison of theoretical MTF for conventional (dashed) and EDI (bold red curve) methods, for a fixed length spectrograph, $500 \mathrm{~nm}$ light, native resolution 5k, goal resolution 50k, and lossless optics. Photon limited signal to noise ratio $(\mathrm{S} / \mathrm{N})$ is plotted against spatial frequency $\rho$ (features per $\mathrm{cm}^{-1}$, having same dimensions as the delay) along the spectrum. To achieve 50k resolution (dashed curve) the conventional slitwidth must be reduced 10x, which can reduce flux 10x for an aberrated or fiber optic source, (and requires focal spot to be 10x smaller, and detector pixel density 10x higher). The $\mathrm{EDI}_{1-7}$ achieves a $50 \mathrm{k}$ net resolution from $5 \mathrm{k}$ native resolution using 7 delays having Gaussian weighted exposure times. Equalization (not shown) would remove the bumps from the EDI curves. The net EDI S/N performance is within $\sqrt{2}$ of the conventional result. The $\mathrm{EDI}_{5-7} \mathrm{MTF}$ (green curve) is a choice of exposures times emphasizing higher frequencies, such as for radial velocity or lineshape asymmetry work, (having same root mean square area as the EDI $1-7$ result). It has more sensitivity than the classic spectrograph for high frequencies.
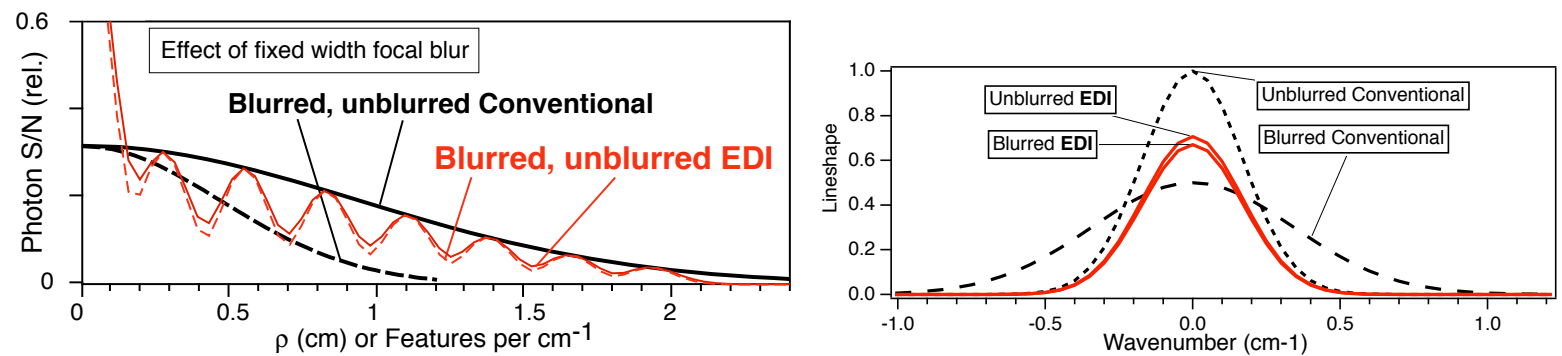

Figure 10. The EDI (red) is dramatically more robust to focal spot or slit aberrations than the conventional spectrograph (black), because EDI data is sensed at specific spatial frequencies defined by a physical delay, not controlled by focal spot size or slit width. Left graph is the effect on MTF (solid to dashed curves) to a fixed width blur which doubles the conventional focal spot, but only increases the EDI spot by $10 \%$ (since it uses a 10x wider slit). The rms average EDI S/N only decreases $\sim 5 \%$ (because $\sqrt{0.9} \simeq 0.95$ ) and its resolution unchanged, while the conventional has its $\mathrm{S} / \mathrm{N}$ for narrow features decimated and its resolution halved. Left graph is corresponding changes to instrument lineshape after the equalization removes bumps in MTF. 
which uses only the 3 highest delays. (The root mean square area under the peaks is conserved, since the same total exposure time is used for all techniques.) This MTF has a greater sensitivity than the classic spectrograph curve at higher frequencies.

\subsection{Instrument qualities synopsis}

The EDI-ISR is a hybrid instrument combining the best qualities of purely dispersive and interferometric (FTS) techniques. Interferometer-like qualities include: compactness, low mass, high resolution, invariance to slit/beam shape/focal blur, need for few pixels, wide simultaneous bandwidth, and a resolution set by maximum delay, not by the grating. Disperser-like qualities include: high photon $\mathrm{S} / \mathrm{N}$ ratio, potential to record broad spectralband snapshots of rapidly changing phenomena, robustness to time dependent sources, and use of sensitive low cost integrating detectors. Unique to EDI is the use of field-widened (angle independent delay) interferometer designs to allow spatially extended sources. These are not practical for the scanning delay of a FTS.

\section{ACKNOWLEDGMENTS}

This material is based upon work supported by the National Science Foundation under Grant No. AST-0505366, AST-096064, NASA Grant NNX09AB38G, and by Lawrence Livermore National Laboratory under Contract DEAC52-07NA27344. Thanks to Ed Wishnow for molecular spectra calculations and valuable advice over the years. Thanks to Palomar Observatory and UC Berkeley Space Sciences staff including Mario Marckwordt, Michael Feuerstein, and Gregory Dalton. Thanks to Triplespec PI Terry Herter and Cornell staff Charles Henderson and Stephen Parshley. Travis Barman provided high-res model stellar spectrum used in TEDI performance simulations.

\section{REFERENCES}

1. H. G. Roe, Titan's atmosphere at high-resolution. PhD thesis, Univ. California, Berkeley, 2002.

2. F. Kerber, G. Nave, and C. J. Sansonetti, "The Spectrum of Th-Ar Hollow Cathode Lamps in the 691-5804 nm region: Establishing Wavelength Standards for the Calibration of Infrared Spectrographs," ApJS 178, pp. 374-381, 2008.

3. D. Erskine, "Combined dispersive/interference spectroscopy for producing a vector spectrum," US Patent 6,351,307, Issued Feb. 26, 2002.

4. D. Erskine, "An externally dispersed interferometer prototype for sensitive radial velocimetry: theory and demonstration on sunlight," PASP 115, pp. 255-269, 2003.

5. D. Erskine, J. Edelstein, M. Feuerstein, and B. Welsh, "High resolution broadband spectroscopy using an externally dispersed interferometer," ApJ 592, pp. L103-L106, 2003.

6. D. J. Erskine and J. Edelstein, "Interferometric resolution boosting for spectrographs," in Ground-based Instrumentation for Astronomy, A. Moorwood and M. Iye, eds., Proc. SPIE 5492, pp. 190-199, 2004.

7. J. Ge, D. Erskine, and M. Rushford, "An externally dispersed interferometer for sensitive Doppler extra-solar planet searches," PASP 114, pp. 1016-1028, 2002.

8. J. Edelstein, D. J. Erskine, J. Lloyd, T. Herter, M. Marckwordt, and M. Feuerstein, "The TEDI instrument for near-IR radial velocity surveys," in Ground-based and Airborne Instrumentation for Astronomy, I. McLean and M. Iye, eds., Proc. SPIE 6269, 2006.

9. J. Edelstein, P. Muirhead, J. Wright, K. Covey, D. Erskine, M. Muterspaugh, J. Lloyd, S. Halverson, M. Marckwordt, and D. Mondo, "Infrared radial velocimetry with TEDI: performance development," in Ground-based and Airborne Instrumentation for Astronomy III, Proc. SPIE 7735, 2010.

10. S. Mahadevan, J. Ge, S. W. Fleming, X. Wan, C. DeWitt, J. C. van Eyken, and D. McDavitt, "An inexpensive field-widened monolithic michelson interferometer for precision radial velocity measurements," PASP 120, pp. 1001-1015, 2008.

11. J. Ge, J. van Eyken, S. Mahadevan, C. DeWitt, S. R. Kane, R. Cohen, A. Vanden Heuvel, S. W. Fleming, P. Guo, G. W. Henry, D. P. Schneider, L. W. Ramsey, R. A. Wittenmyer, M. Endl, W. D. Cochran, E. B. Ford, E. L. Martín, G. Israelian, J. Valenti, and D. Montes, "The first extrasolar planet discovered with a new-generation high-throughput Doppler instrument," ApJ 648, pp. 683-695, 2006. 
12. P. S. Muirhead, J. Edelstein, D. J. Erskine, J. T. Wright, M. W. Muterspaugh, K. R. Covey, E. H. Wishnow, K. Hamren, P. Andelson, D. Kimber, T. Mercer, S. P. Halverson, A. Vanderburg, D. Mondo, A. Czeszumska, and J. P. Lloyd, "Precise stellar radial velocities of an $\mathrm{M}$ dwarf with a Michelson interferometer and a medium-resolution near-infrared spectrograph," PASP 123(904), pp. 709-724, 2011.

13. J. C. Wilson, C. P. Henderson, T. L. Herter, K. Matthews, M. F. Skrutskie, J. D. Adams, D.-S. Moon, R. Smith, N. Gautier, M. Ressler, B. T. Soifer, S. Lin, J. Howard, J. LaMarr, T. M. Stolberg, and J. Zink, "Mass producing an efficient NIR spectrograph," in Ground-based Instrumentation for Astronomy, A. Moorwood and M. Iye, eds., Proc. SPIE 5492, pp. 1295-1305, 2004.

14. R. Beer, Remote Sensing by Fourier Transform Spectrometry, John Wiley, New York, 1992.

15. B. B. Behr, A. R. Hajian, A. T. Cenko, M. Murison, R. S. McMillan, R. Hindsley, and J. Meade, "Stellar astrophysics with a dispersed Fourier transform spectrograph. I. Instrument description and orbits of singlelined spectroscopic binaries," ApJ 705, pp. 543-553, 2009. 